\title{
Critérios para a tomada de decisão em obras rodoviárias sustentáveis
}

\author{
Criteria for decision making in sustainable road works
}






\section{Introdução}

A priorização constitui uma sofisticação no processo de tomada de decisão em obras rodoviárias, pois é capaz de determinar preferências de intervenção de acordo com cenários estabelecidos pelo usuário, tendo como objetivo principal a maximização dos benefícios à sociedade.

Contudo, a maioria dos procedimentos para tomada de decisão no meio rodoviário utiliza métodos de programação matemática, incluindo programação linear, inteira e dinâmica, em que são desenvolvidas soluções otimizadas de acordo com um objetivo estabelecido, que em grande parte é o econômico (HDM-4-b, 2000). Os mais difundidos na análise de estratégias de manutenção de pavimentos são o da Enumeração Total (adotada pelo modelo EBM-HS do HDM-III, que escolhe a alternativa, em um elenco, com maior valor presente líquido total) e da análise do Valor Incremental Benefício/Custo (adotado no HDM-4 - escolhe a alternativa com a maior razão entre o valor presente da rede e custos) (PORATH, 2002; HDM-4-b, 2000).

Mais recentemente, o HDM-4-b (2000) indicou a análise multicriterial quando, além das análises que podem ser transcritas em termos monetários, se deseja inserir critérios difíceis de serem mensurados monetariamente (ex.: impactos ambientais negativos, desenvolvimento social). Por meio dessa análise, a priorização pode ser realizada utilizando-se fatores que seriam difíceis de ser avaliados de uma só vez (maximizar benefício/custo, maximizar índice de condição geral do pavimento, minimizar impacto negativo ao meio ambiente, maximizar o desenvolvimento econômico local etc.). Nesse tipo de análise a escolha não vai apontar uma melhor solução para um objetivo particular, mas uma solução que atenda da melhor forma possível a todos os critérios analisados no sistema.

Uma das ferramentas mais sofisticadas para análise multicriterial é o Método PROMETHEE (BRANS et al., 1984). Ele pertence à Família Baseada em Relações Hierárquicas, em que a ordem preferencial da variável de cada critério analisado é normalizada por uma função de preferência. $\mathrm{O}$ tomador de decisão deve indicar a necessidade de maximizar ou minimizar os valores de cada critério dentro de uma função que determinará a preferência, e a mesma definirá a hierarquização das alternativas analisadas (ZUFFO, 1998).

O método PROMETHEE (algumas de suas versões são o PROMETHEE I e o PROMETHEE II) já é amplamente utilizado na macroeconomia para a escolha de tipos de estratégias de desenvolvimento, na escolha da localização de implantação de indústrias e localização de implantação de obras de infraestrutura energética (hidroelétricas) e de abastecimento d'água (reservatórios). Na microeconomia é usada na escolha de protótipos para produção industrial, alocação de microempresas etc.

Nesse contexto, o Método de Análise Multicriterial PROMETHEE II pode ser avaliado como uma proposta de ferramenta para o suporte à decisão na manutenção de rodovias. Neste trabalho, para a avaliação desse método, são utilizados critérios das categorias técnica, econômica, social, ambiental e de tráfego na hierarquização de 23 trechos de rodovias diferentes do estado da Paraíba. Objetivase não só o atendimento aos níveis mínimos de serventia estabelecidos para a malha rodoviária, mas também a avaliação das necessidades atuais da sociedade servida e a promoção de uma alternativa para apoio ao desenvolvimento sustentável.

\section{Análise multicriterial}

\section{Considerações iniciais}

Os problemas que envolvem múltiplos critérios são distinguidos em dois grupos diferentes. No primeiro grupo, Contínuo, o conjunto de alternativas considerado por parte do tomador de decisão é infinito, dado o caráter matemático contínuo de soluções factíveis. No segundo grupo, Discreto, o conjunto de alternativas considerado por parte do tomador de decisão é finito e normalmente pouco elevado (RÓDENAS; BARBERIS, 2002).

Os problemas de engenharia, sobretudo os de escolha de alternativas em pavimentação, se encaixam bem no Grupo Discreto de problemas multicriteriais, pelo fato de as soluções de intervenção apontadas terem de atender a um desempenho mínimo especificado.

A solução de problemas multicriteriais dentro do Grupo Discreto pode ser realizada com ferramentas conhecidas como Métodos de Preferências (utiliza funções de preferência em que, a cada par de alternativas, são associados um índice de preferência e um índice de indiferença), que classifica uma alternativa como melhor do que outra. A concepção desses métodos foi realizada por um grupo de pesquisadores franceses em meados dos anos 1960, sendo bem aceita em todo 
o mundo como Método de Análise Multicriterial Discreto (RÓDENAS; BARBERIS, 2002).

$\mathrm{O}$ primeiro pesquisador que utilizou o método de análise multicriterial por preferências foi o francês Bernard Roy, através do método ELECTRE (Elimination Et Choix Traduisant la Realité) (RÓDENAS; BARBERIS, 2002).

Entre os métodos mais recentes de análise multicriterial por preferências destaca-se o método PROMETHEE (Preference Ranking Organization METHod for Enrichment Evaluations). Esse método foi desenvolvido por Jean Pierre Brans e Bertrand Mareschal (BRANS et al., 1984), da Université Livre de Brussels (Bélgica), sob orientação de Bernard Roy.

Esses dois métodos, especialmente o PROMETHEE, têm hoje grande repercussão mundial e ficaram conhecidos como métodos da Escola Europeia de Decisão Multicriterial, ou mesmo Escola Franco-Belga. Este último é, provavelmente, o método de Análise Multicriterial mais utilizado em todo o mundo, muito por ajudar a resolver, de forma matematicamente simplificada e sem incompatibilidades, problemas de difícil compreensão nas mais diversas áreas, como:

(a) recrutamento em RH (vendedores, peritos, gerentes superiores etc.);

(b) classificação preferencial de projetos (financiamento, empréstimos, priorização etc.);

(c) planejamento estratégico (aquisição, fusão, expansão, obras de infraestrutura etc.);

(d) monitoramento de desempenho (garantia da qualidade, padrões técnicos etc.); e

(e) tomada de decisão do grupo (óticas diferentes das partes interessadas etc.).

\section{Método PROMETHEE}

O PROMETHEE é um método de preferência que fornece ao tomador de decisão uma estrutura preferencial entre alternativas discretas (PORTO, 1997).

Sendo $A$ o conjunto de alternativas, para cada ação (alternativa) $a_{i} \in A, i=1, \ldots n, f_{j}\left(a_{i}\right)$ é uma avaliação dessa ação segundo o critério $j, j=1, \ldots . k$. Essas avaliações podem ser representadas na matriz $M$ (LOPES, 2005):

$M=\left[\begin{array}{cccc}f_{1}\left(a_{1}\right) & f_{2}\left(a_{1}\right) & \ldots & f_{k}\left(a_{1}\right) \\ f_{1}\left(a_{2}\right) & f_{2}\left(a_{2}\right) & \ldots & f_{k}\left(a_{2}\right) \\ \vdots & \vdots & & \\ f_{1}\left(a_{n}\right) & f_{2}\left(a_{n}\right) & \ldots & f_{k}\left(a_{n}\right)\end{array}\right]$
A partir de $M$, uma avaliação aos pares é efetuada, seguindo o algoritmo (BRANS et al., 1984):

(a) especificar para cada critério $f_{j}$ uma função de preferência generalizada $\left(\mathrm{P}_{\mathrm{j}}\right)$ tal que:

$P_{j}: A \times A \rightarrow[0,1]$.

Eq. 2

Na comparação entre as alternativas quaisquer $a_{r} \mathrm{e}$ $a_{s}$, pertencentes ao conjunto $A$, tem-se:

$P_{j}\left(a_{r}, a_{s}\right)=P(x)=P\left[f\left(a_{r}\right)-f\left(a_{s}\right)\right]$, que representa a intensidade com que a alternativa (ou ação) $a_{r}$ é preferível a $a_{s}$ segundo o critério $j$, tal que:

- se $P(x)=0$, não há preferência de $a_{r}$ em relação a $a_{s}$;

- se $P(x) \cong 0$, há fraca preferência de $a_{r}$ em relação a $a_{s}$;

- se $P(x) \cong 1$, há forte preferência de $a_{r}$ em relação

a $a_{s} ; \mathrm{e}$

- se $P(x)=1$, há total preferência de $a_{r}$ em relação

a $a_{s}$.

Se o critério precisa ser maximizado, usa-se $x=f\left(a_{r}\right)-f\left(a_{s}\right)$ para definir a função de preferência. No caso de minimização do critério, a função de preferência será dada por $x=f\left(a_{s}\right)-f\left(a_{r}\right)$.

Brans, Vincke e Mareschal (1986) determinaram seis tipos de função de preferência. As funções e representações gráficas $-H(x)$ - são apresentadas na Tabela 1. O avaliador ainda pode modelar outras funções de preferência de interesse. O significado de cada função é:

- na função de preferência Linear, há indiferença entre as alternativas $a_{r}$ e $a_{s}$ somente se $f\left(a_{r}\right)=f\left(a_{s}\right)$. Quando as avaliações são diferentes, o avaliador tem preferência total pela alternativa que possui melhor avaliação;

- na função de preferência $U$-Shape, as alternativas são indiferentes sob o ponto de vista do avaliador se não excedem o limite de indiferença $q$. Caso contrário, há preferência total pela alternativa $a_{r}$;

- na função de preferência $V$-Shape, $r$ é o limite de preferência total, e se a diferença entre as alternativas for menor do que esse limite, então a preferência pela alternativa $a_{r}$ aumenta linearmente com a diferença $x$. Quando a diferença é maior do que $r$, há preferência total pela alternativa $a_{r}$;

- na função de preferência Level, o avaliador deve definir o limite de indiferença $q$ e o limite de preferência $r$ tal que: se $x$ é menor do que $q$, então há indiferença entre as alternativas; se $x$ está entre $q$ e $r$, há fraca preferência (1/2) por $a_{r}$; e se $x$ é 
maior do que $r$, então a alternativa $a_{r}$ é preferível à alternativa $a_{s}$;

- na função de preferência $V$-Shape $I$, o avaliador considera que sua preferência aumenta linearmente da indiferença para a total preferência entre os limites $q$ e $r$; e

- na função de preferência Gaussian, é necessário apenas que o avaliador defina o parâmetro $s$, que indica o valor a partir do qual há mudança na concavidade na curva de preferência.

(b) definir os pesos $\alpha_{j}(j=1,2, \ldots, \mathrm{n})$, que são as medidas de importância de cada critério. Essa avaliação é feita pelos tomadores de decisão ou especialistas. Pode ocorrer que os pesos sejam todos iguais, se os critérios tiverem a mesma importância. (c) calcular para todos os pares de alternativas o índice de preferência ponderada global $-\pi\left(a_{r}, a_{s}\right)$, que indica o percentual de preferência da alternativa $a_{r}$ em relação à alternativa $a_{s}$, levando em consideração os pesos atribuídos a cada critério (Equação 1).

$\pi\left(a_{r}, a_{s}\right)=\sum_{j=1}^{n} \alpha_{j} P_{j}\left(a_{r}, a_{s}\right)$

Onde:

$\sum_{j=1}^{n} \alpha_{j}=1$

Eq. 4

$0 \leq \pi\left(a_{r}, a_{s}\right) \leq 1 \quad \forall \quad a_{r}, a_{s} \in A$

Eq. 5

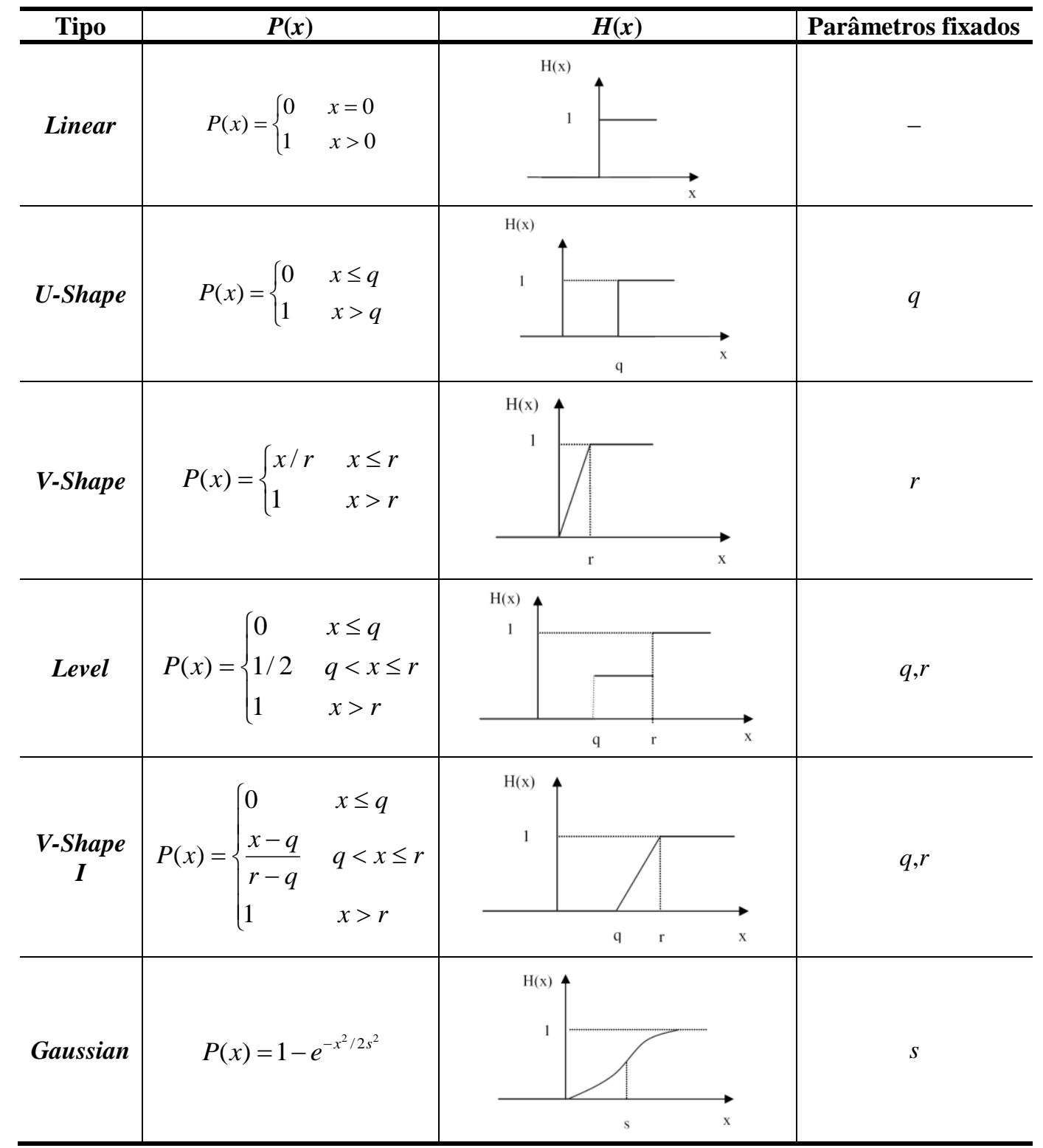

Tabela 1 - Funções de preferência do método PROMETHEE descritas por Brans, Vincke e Mareschal (1986) 
(d) calcular o fluxo de importância positivo, que representa a média de preferência de qualquer alternativa $a_{r}$ sobre todas as alternativas do conjunto $A$ (Equação 6):

$\phi^{+}\left(a_{r}\right)=\frac{1}{n-1} \cdot \sum_{x \in A}^{n} \pi\left(a_{r}, x\right)$

Onde:

$\phi^{+}: A \rightarrow[0,1]$

quanto maior $\phi^{+}\left(a_{r}\right)$, melhor a alternativa;

(e) calcular o fluxo de importância negativo, que representa a média de preferência de todas as alternativas sobre a alternativa $a_{r}$ (Equação 7):

$\phi^{-}\left(a_{r}\right)=\frac{1}{n-1} \cdot \sum_{x \in A}^{n} \pi\left(x, a_{r}\right)$

Eq. 7

Onde:

$\phi^{-}: A \rightarrow[0,1]$

quanto menor $\phi^{-}\left(a_{r}\right)$, melhor a alternativa; e

(f) como saída do algoritmo, tem-se a

classificação completa (PROMETHEE II).

O PROMETHEE II consiste em hierarquizar as ações seguindo uma ordem decrescente. Generaliza o conceito da qualificação, em que não há "incompatibilidades", fornecendo uma ordenação completa e única. Essa classificação completa das alternativas é realizada através do fluxo de importância líquido de preferência $(\phi)$ calculado pela diferença entre os fluxos de importância positivo e negativo (Equação 8), como se segue: $\phi\left(a_{r}\right)=\phi^{+}\left(a_{r}\right)-\phi^{-}\left(a_{r}\right)$

Eq. 8

Onde:

$\phi: A \rightarrow R$

$a_{r}$ é preferível à $a_{s}\left(a_{r} P^{I I} a_{s}\right)$ se: $\phi\left(a_{r}\right)>\phi\left(a_{s}\right)$; e

$a_{r}$ é indiferente à $a_{s}\left(a_{r} I^{I I} a_{s}\right)$ se: $\phi\left(a_{r}\right)=\phi\left(a_{s}\right)$.

O Método PROMETHEE é uma tentativa de estabelecer, de forma natural, a estrutura de preferência do tomador de decisão. Tem-se mostrado um método fácil de ser aplicado, envolvendo ao mesmo tempo uma análise mais completa e explícita (PORTO, 1997).

\section{Critérios adotados}

\section{Características dos trechos rodoviários}

Os trechos de rodovias analisados neste trabalho fazem parte da malha rodoviária do estado da Paraíba, localizado na parte leste da Região Nordeste do Brasil. Esse estado apresenta grande heterogeneidade em termos de desenvolvimento, com a região litorânea bem mais desenvolvida que o interior do estado, o que motivou a avaliação dessa metodologia como também promotora de desenvolvimento. A extensão total de rodovias estaduais pavimentadas na Paraíba é de $2.177,00$ $\mathrm{km}$. Devido à grande quantidade de trechos rodoviários estaduais existentes nesse estado (234), as análises foram realizadas em uma amostra aleatória de $10 \%$ do total (23 trechos), com extensão de $268 \mathrm{~km}$. A distribuição espacial dos trechos escolhidos encontra-se na Figura 1 (ALBUQUERQUE, 2007).

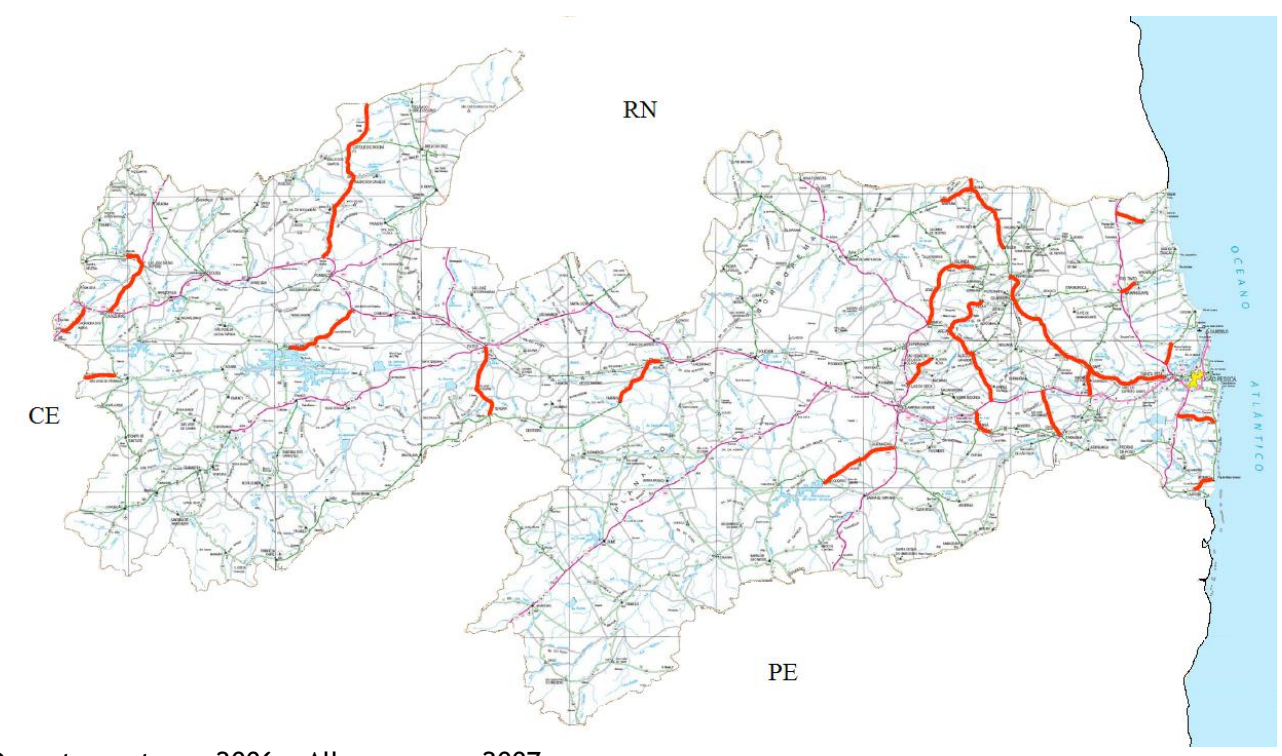

Fontes: Departamento..., 2006 e Albuquerque, 2007.

Figura 1 - Mapa esquemático com localização dos trechos rodoviários utilizados na priorização 
As análises foram processadas de acordo com a situação atual de cada trecho rodoviário. Primeiramente se realizou um diagnóstico de rede, verificando a necessidade presente de intervenção de cada trecho rodoviário. Levantou-se também o custo de manutenção $(C M)$, para restabelecer as boas condições ao pavimento.

Os critérios adotados neste trabalho foram selecionados de acordo com dois fatores: disponibilidade de dados e facilidade de aplicação. Esses critérios estão descritos nos itens a seguir, levando-se em consideração as seguintes categorias: técnica, econômica, social, ambiental e de tráfego.

\section{Critérios técnicos}

Três critérios técnicos foram utilizados na análise. Suas descrições são as seguintes:

(a) Índice de Condição do Pavimento $\left(P C I_{\text {atual }}\right)$ : parâmetro que determina a condição atual da superfície do pavimento, calculado considerando o valor de 100 (pavimento em perfeito estado) deduzido de valores correspondentes ao peso e extensão dos defeitos até o valor mínimo de 0 (pavimento em péssima condição) (SHAHIN, 1994);

(b) Vida Residual: tempo restante em anos para que o $P C I$ do trecho rodoviário seja igual a 40;

(c) Número Anual de Repetições do Eixo Padrão $-N_{\text {ano }}$ : representa a equivalência de eixos simples de rodas duplas de 8,2 t que trafega no pavimento em 1 ano (IPR-723, 2006); e

(d) Custo de Manutenção (R\$): valor financeiro necessário para a reabilitação do pavimento.

\section{Critérios econômicos}

Para a análise dos critérios econômicos empregouse o banco de dados existente na página da web do IBGE (IBGE, 2007a) sobre os municípios brasileiros. Foram utilizados os seguintes critérios, que caracterizavam os municípios servidos diretamente por cada trecho rodoviário analisado:

(a) PIB Agropecuário (R\$): Produto Interno Bruto Agropecuário;

(b) Potencial Agrícola: foi utilizado o mapa da Figura 2 para a identificação da região agrícola em que cada trecho rodoviário está inserido. A escala adotada na análise varia de Boa (10) a

Desfavorável (1), conforme conceitos identificados na Figura 2;

(c) PIB Industrial (R\$): Produto Interno Bruto Industrial; e

(d) Via de Acesso Turístico: um trecho rodoviário considerado via turística recebe pontuação 2 , caso contrário recebe pontuação 1 .

\section{Critérios sociais}

$\mathrm{Na}$ análise dos critérios sociais também foi usado o banco de dados existente na página da web do IBGE (IBGE, 2007b) sobre os municípios brasileiros. Os critérios que caracterizavam os municípios servidos diretamente por cada trecho rodoviário analisado são:
(a) População (número de habitantes);
(b) Índice de Desenvolvimento Humano - IDH; e
(c) Produto Interno Bruto por Habitante: PIB per capita (R\$/hab.).



Fonte: IBGE, 2007c.

Figura 2 - Mapeamento do potencial agrícola do estado da Paraíba 


\section{Critérios ambientais}

Os critérios ambientais analisados foram: Necessidade de Extração de Recursos Minerais; e Proximidade de Unidades de Proteção.

Para a análise da Necessidade de Extração de Recursos Minerais foram atribuídas pontuações relativas ao nível de utilização de recursos minerais em cada tipo de intervenção apontada na Árvore de Decisão para Manutenção de Pavimentos (Figura 3). Para a situação de "Nada a Fazer" não houve necessidade de extração de recursos minerais (pontuação 0). Para a "Conserva" foi considerada uma necessidade "Muito Baixa" (pontuação 1) de extração de recursos minerais; para aplicação de "Lama Asfáltica", "Baixa" (pontuação 2); para "Correções Localizadas ou Recapeamento", "Moderada" (pontuação 3); "Restauração", "Alta" (pontuação 4); e para "Reconstrução da Pista", "Muito Alta" (pontuação 5).

Quanto à Proximidade de Unidades de Proteção, foi utilizada a distância em quilômetros do Trecho
Rodoviário até a Unidade de Proteção Ambiental (Figura 4) mais próxima. Entendendo-se que, quanto menor for essa distância, maior será a vulnerabilidade da Unidade de Proteção Ambiental, então se deseja que essa distância seja a maior possível.

\section{Critérios de tráfego}

Foram adotados três critérios relativos ao tráfego. O primeiro foi o Volume Diário Médio (VDM) de veículos que trafegam no trecho rodoviário; o segundo, a Frota Disponível nas Cidades; e o terceiro, a Extensão do Segmento.

No caso do VDM e Extensão do Segmento, foram utilizados os dados fornecidos pelo Departamento de Estradas de Rodagem do Estado da Paraíba (DER-PB). Para a Frota Disponível nas Cidades, foi utilizado o banco de dados existente na página da web do IBGE (IBGE, 2007a) sobre os municípios brasileiros, calculando-se o somatório das frotas dos municípios que são ligados pelo trecho rodoviário analisado.

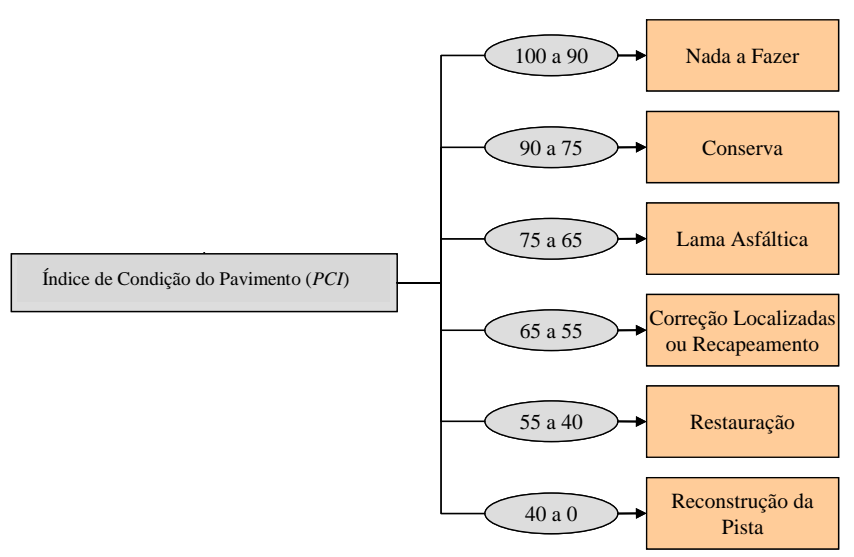

Figura 3 - Árvore de decisão para manutenção de pavimentos

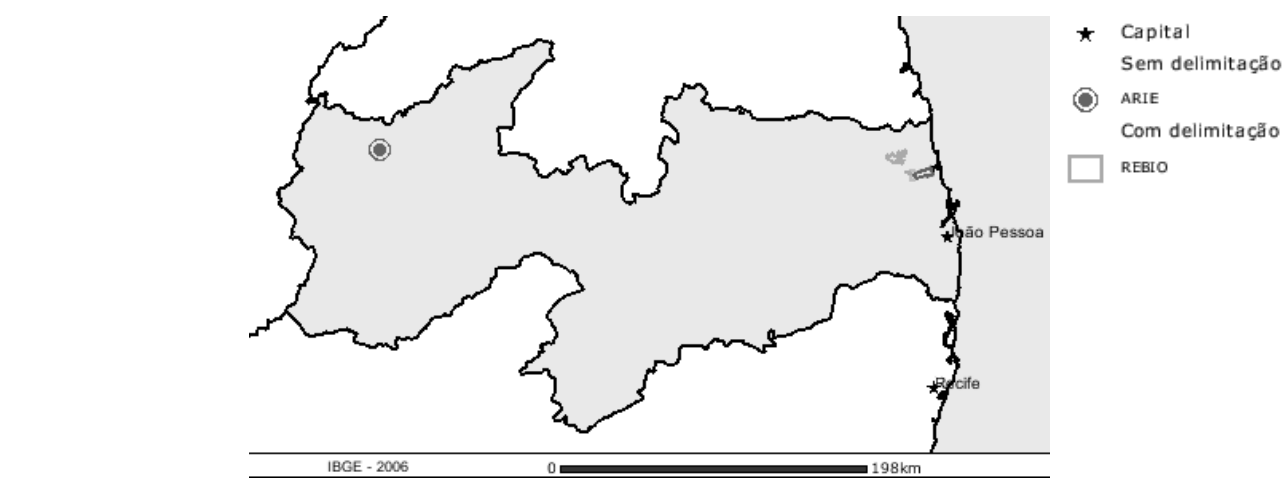

Fonte: IBGE, 2007c.

Figura 4 - Mapeamento das unidades de proteção ambiental no estado da Paraíba 


\section{Cenários analisados}

A Priorização foi realizada aplicando-se a ferramenta de Análise Multicriterial PROMETHEE II, que serve para hierarquizar os trechos rodoviários quanto à necessidade de intervenção. Três cenários diferentes foram aplicados para proceder às análises de priorização. Os cenários foram estruturados com o objetivo único de proporcionar a comparação de técnicas de priorização normalmente adotadas na engenharia rodoviária e a técnica utilizando multicritérios, conforme descrito a seguir.

(a) o Cenário 01, chamado de Construção Sustentável, consiste em uma análise multicritério. Ele foi estruturado utilizando o método
PROMETHEE II em planilha eletrônica. Os critérios de categoria técnica têm peso total de $60 \%$ na análise, e cada um dos conjuntos de critérios das categorias econômica, social, ambiental e de tráfego tem peso de $10 \%$. Na definição dos pesos foi dada maior ênfase aos critérios técnicos devido a eles serem considerados como de grande importância pelo DER-PB, porém o tomador de decisão tem liberdade de definir a ótica de seu interesse. O resumo dos critérios utilizados e suas correspondentes funções de preferência e pesos estão apresentados na Figura 5;

(b) o Cenário 02 compreende uma classificação direta crescente do $P C I$, método simples e bastante comum no meio rodoviário, no qual o trecho com menor $P C I$ tem maior prioridade; e



Figura 5 - Critérios, funções de preferência e pesos para priorização do Cenário 01 
(c) o Cenário 03 consiste na análise

Benefício/Custo, comumente utilizada em avaliações econômicas para obras rodoviárias, na qual, quanto maior a relação, maior é a prioridade de intervenção do trecho rodoviário. $\mathrm{O}$ método de cálculo da razão Benefício/Custo seguiu o seguinte procedimento (Equação 9) (NHI, 1998):

Benefício/custo $=\left[\left(P C I_{0}-P C I_{\text {atual }}\right) V D M\right] / C M(\mathrm{R} \$) \quad$ Eq. 9

Onde:

(a) $C M$ é o custo de manutenção do pavimento associado ao valor do $P C I_{\text {atual }}$ do trecho rodoviário;

(b) $P C I_{\text {atual }}$ é $P C I$ no ano de análise;

(c) $P C I_{0}$ é o $P C I$ calculado para a situação logo após a aplicação da intervenção sugerida na Árvore de Decisão da Figura 3; e

(d) VDM é o Volume Diário Médio de veículos que circula no trecho rodoviário.

\section{Resultados obtidos}

A Tabela 2 apresenta as características dos trechos rodoviários para cada critério utilizado nos cenários analisados.

$\mathrm{Na}$ avaliação preliminar dos dados, constatou-se que as melhores condições socioeconômicas se encontram em trechos rodoviários fora de regiões de clima seco ou semiárido, ou seja, na faixa litorânea do estado. O tráfego também é maior nesses mesmos trechos rodoviários.

O detalhamento de cálculos da análise Benefício/Custo encontra-se na Tabela 3. Nota-se que os trechos rodoviários estão classificados decrescentemente, de acordo com os resultados Benefício/Custo. Os resultados dessa relação são bastante influenciados pela medida de manutenção escolhida e pelo VDM. A medida de manutenção determinará o PCI futuro, que influencia no benefício adquirido e no custo da obra. O VDM funciona como multiplicador de um benefício individual. Várias medidas de manutenção poderiam ser selecionadas como possíveis, porém foi necessário fixar uma medida para fins de comparação entre os Cenários 01 e 03. A Tabela 4 apresenta o comparativo entre resultados de priorização dos Cenários 01, 02 e 03.

Observa-se nas hierarquizações apresentadas na Tabela 4 que os cenários analisados não mantiveram correspondência entre si. As priorizações foram bem distintas, principalmente quando se observam os três primeiros trechos rodoviários na hierarquização de cada método.
No Cenário 02 (Classificação do $P C I$ ) o trecho rodoviário prioritário foi o PB-393/003. Contudo, devido a sua menor representação econômica e de tráfego, o Cenário 01 o classificou como $8^{\circ} \mathrm{em}$ prioridade, e no Cenário 03 ele ficou apenas em $19^{\circ}$.

O trecho rodoviário $\mathrm{PB}-041 / 007$ foi o prioritário no Cenário 03, Benefício/Custo, enquanto no Cenário 01 ele foi o $13^{\circ}$, e no Cenário 02 ele foi o $11^{\circ}$.

O Cenário 01, Construção Sustentável, colocou o trecho PB-073/001 como prioritário na hierarquização, devido ao Custo de Manutenção ser relativamente baixo quando comparado aos demais trechos rodoviários analisados. Além disso, as características sociais da região servida exigem boas condições da rodovia para proporcionar desenvolvimento, com população atendida considerável (53.562 pessoas), baixo IDH $(0,556)$ e PIB per capita (R\$ 2.520,06). Ainda, a necessidade de extração de recursos minerais para a realização da intervenção é baixa (1), e esse trecho é um dos mais distantes de unidades de proteção ambiental $(60,29 \mathrm{~km})$. Esse trecho rodoviário também possui um tráfego diário considerado intenso, segundo classificação do DNIT (VDM igual a 3.026) (IPR-723, 2006).

O Cenário 01 proposto para aplicação do método PROMETHEE II conduziu a um resultado próximo ao desejado pelo DER-PB, devido aos critérios técnicos reunirem $60 \%$ de peso na decisão. Os interesses diversos (representando os diversos setores da sociedade) se distribuíram nos demais $40 \%$ dos pesos. O Cenário 01 estabelecido atuou também como mecanismo para promover desenvolvimento. Exemplo disso foi a escolha do trecho PB-004/007 como $2^{\circ}$ na priorização, trecho localizado em região com índices sociais insatisfatórios (IDH igual a 0,554), enquanto no Cenário 03 este trecho teve a $16^{\mathrm{a}}$ colocação. O Cenário 01 ainda identificou o trecho rodoviário PB-018/001 como $3^{\circ}$ na hierarquização. Este trecho apresentava PCI abaixo de 75 (abaixo do limite para manutenção) e características econômicas relevantes (alto PIB industrial e via de acesso turístico).

Apesar de os Cenários 02 e 03 tratarem de critérios técnicos, seus resultados de priorização apresentaram grandes diferenças, as quais se devem principalmente ao Cenário 02 avaliar uma situação estritamente atual $\left(P C I_{\text {atual }}\right)$, enquanto o Cenário 03 avalia uma projeção de situação futura (PCI futuro e custos futuros). O método de previsão do $P C I$ é determinante para o sucesso da análise quando se utiliza o Cenário 03. 


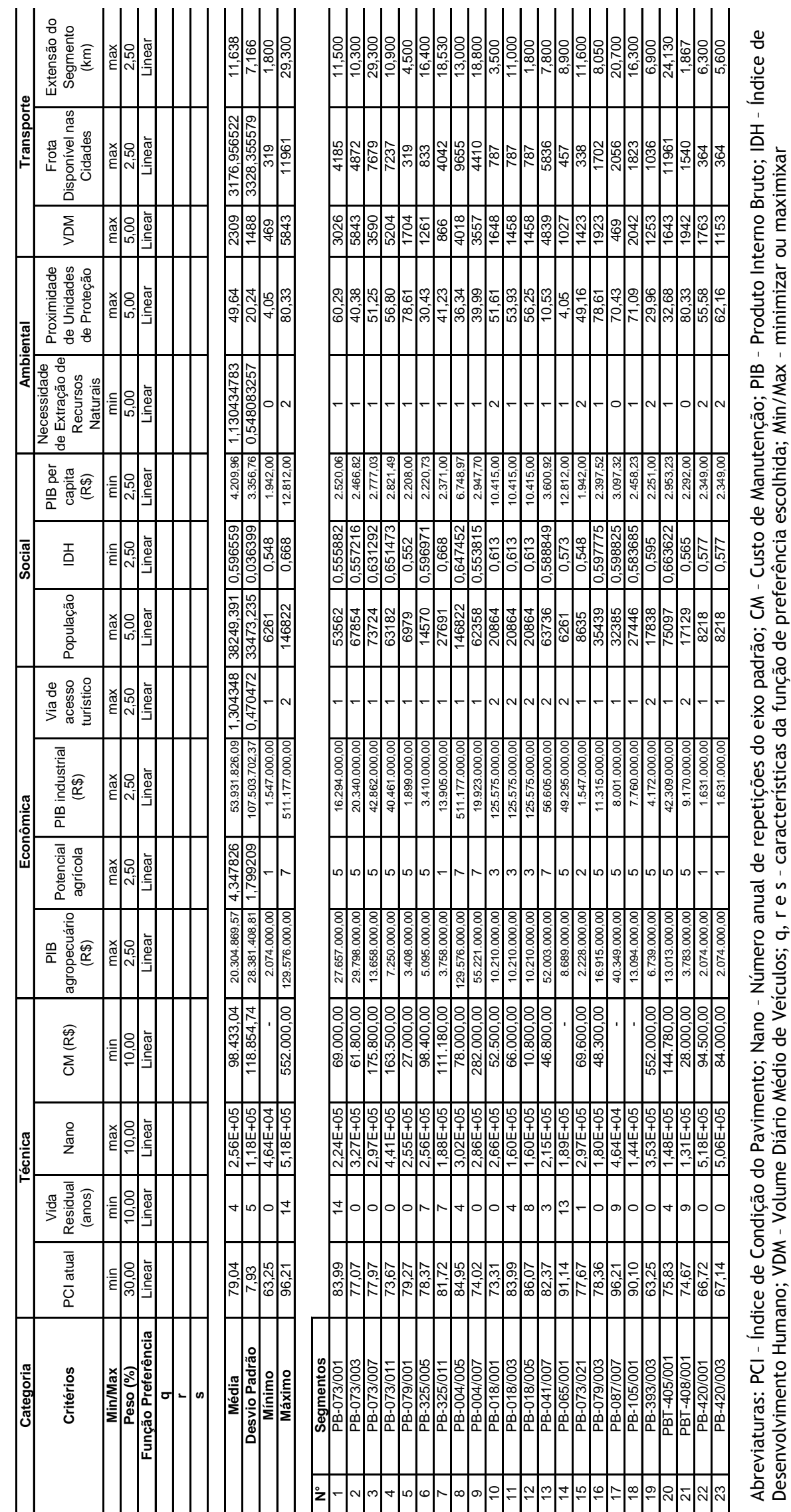

Tabela 2 - Planilha de dados para análise multicriterial 


\begin{tabular}{l|c|c|c|c|c}
\hline Trecho & Custo & PCI $_{\text {atual }}$ & VDM & PCI $_{\boldsymbol{o}}$ & Benefício/Custo \\
\hline PB-041/007 & $46.800,00$ & 82,4 & 4.839 & 97,8 & 1,5953 \\
\hline PB-073/003 & $61.800,00$ & 77,1 & 5.843 & 88,7 & 1,1016 \\
\hline PB-018/005 & $10.800,00$ & 86,1 & 1.458 & 94,0 & 1,0668 \\
\hline PB-004/005 & $78.000,00$ & 85,0 & 4.018 & 97,9 & 0,6683 \\
\hline PB-079/003 & $48.300,00$ & 78,4 & 1.923 & 93,1 & 0,5859 \\
\hline PB-079/001 & $27.000,00$ & 79,3 & 1.704 & 88,1 & 0,5560 \\
\hline PB-073/011 & $163.500,00$ & 73,7 & 5.204 & 89,4 & 0,4997 \\
\hline PBT-408/001 & $28.000,00$ & 74,7 & 1.942 & 81,5 & 0,4731 \\
\hline PB-018/001 & $52.500,00$ & 73,3 & 1.648 & 86,4 & 0,4106 \\
\hline PB-073/021 & $69.600,00$ & 77,7 & 1.423 & 96,6 & 0,3870 \\
\hline PB-420/001 & $94.500,00$ & 66,7 & 1.763 & 85,6 & 0,3527 \\
\hline PB-420/003 & $84.000,00$ & 67,1 & 1.153 & 85,6 & 0,2537 \\
\hline PB-073/007 & $175.800,00$ & 78,0 & 3.590 & 88,5 & 0,2160 \\
\hline PB-018/003 & $66.000,00$ & 84,0 & 1.458 & 91,9 & 0,1746 \\
\hline PB-073/001 & $69.000,00$ & 84,0 & 3.026 & 87,8 & 0,1656 \\
\hline PB-004/007 & $282.000,00$ & 74,0 & 3.557 & 86,3 & 0,1552 \\
\hline PBT-405/001 & $144.780,00$ & 75,8 & 1.643 & 86,1 & 0,1166 \\
\hline PB-325/005 & $98.400,00$ & 78,4 & 1.261 & 85,0 & 0,0846 \\
\hline PB-393/003 & $552.000,00$ & 63,3 & 1.253 & 85,8 & 0,0511 \\
\hline PB-325/011 & $111.180,00$ & 81,7 & 866 & 86,6 & 0,0378 \\
\hline PB-065/001 & - & 91,1 & 1.027 & 98,6 & 0,0000 \\
\hline PB-087/007 & - & 96,2 & 469 & 98,8 & 0,0000 \\
\hline PB-105/001 & - & 90,1 & 2.042 & 101,9 & 0,0000 \\
\hline Deta/hamento & - & - & &
\end{tabular}

Tabela 3 - Detalhamento de cálculos da análise Benefício/Custo

\begin{tabular}{|c|c|c|c|}
\hline Hierarquização & Cenário 01 & Cenário 02 & Cenário 03 \\
\hline 1 & PB-073/001( $\left.{ }^{1}\right)$ & PB-393/003( ${ }^{(1)}$ & PB-041/007(*) \\
\hline 2 & PB-004/007( $\left.{ }^{2}\right)$ & PB-420/001( $\left.{ }^{\mathrm{II}}\right)$ & PB-073/003(**) \\
\hline 3 & PB-018/001( $\left.{ }^{3}\right)$ & PB-420/003( $\left.{ }^{\mathrm{III}}\right)$ & PB-018/005(***) \\
\hline 4 & PB-420/001( $\left(^{\mathrm{II}}\right)$ & PB-018/001( $\left.{ }^{3}\right)$ & PB-004/005 \\
\hline 5 & PB-073/011 & PB-073/011 & PB-079/003 \\
\hline 6 & PB-420/003(III) & PB-004/007( $\left.{ }^{2}\right)$ & PB-079/001 \\
\hline 7 & PB-073/003(**) & PBT-408/001 & PB-073/011 \\
\hline 8 & PB-393/003( $\left.{ }^{\mathrm{I}}\right)$ & PBT-405/001 & PB-073/001( $\left.{ }^{1}\right)$ \\
\hline 9 & PB-079/003 & PB-073/001( $\left.{ }^{1}\right)$ & PBT-408/001 \\
\hline 10 & PBT-408/001 & PB-073/003(**) & PB-018/001( $\left.{ }^{3}\right)$ \\
\hline 11 & PB-041/007(*) & PB-073/021 & PB-073/021 \\
\hline 12 & PBT-405/001 & PB-073/007 & PB-420/001( $\left({ }^{\mathrm{II}}\right)$ \\
\hline 13 & PB-073/021 & PB-079/003 & PB-420/003( ${ }^{\text {III }}$ ) \\
\hline 14 & PB-073/007 & PB-325/005 & PB-073/007 \\
\hline 15 & PB-004/005 & PB-079/001 & PB-018/003 \\
\hline 16 & PB-079/001 & PB-325/011 & PB-004/007 $\left(^{2}\right)$ \\
\hline 17 & PB-018/003 & PB-041/007(*) & PBT-405/001 \\
\hline 18 & PB-325/005 & PB-018/003 & PB-325/005 \\
\hline 19 & PB-018/005(***) & PB-004/005 & PB-393/003( $\left.{ }^{1}\right)$ \\
\hline 20 & PB-105/001 & PB-018/005(***) & PB-325/011 \\
\hline 21 & PB-325/011 & PB-105/001 & PB-065/001 \\
\hline 22 & PB-065/001 & PB-065/001 & PB-087/007 \\
\hline 23 & PB-087/007 & PB-087/007 & PB-105/001 \\
\hline
\end{tabular}

Legenda:

$\left({ }^{1}\right),\left({ }^{2}\right)$ e $\left({ }^{3}\right)$ : primeiro, segundo e terceiro na priorização do Cenário 01;

('), (") e ('II): primeiro, segundo e terceiro na priorização do Cenário 02; e

("), (") e ("*): primeiro, segundo e terceiro na priorização do Cenário 03.

Tabela 4 - Comparativo de métodos de priorização na Paraíba 
Toda essa estrutura lógica, reunindo critérios técnicos, econômicos, sociais, ambientais e de tráfego, para análise de priorização só se tornou possível com a formalização por método multicriterial. Contudo, a distribuição de pesos que foi adotada para o processamento das análises poderia ser totalmente modificada, de acordo com a ótica de análise desejada pelo tomador de decisão.

É importante observar que, embora sejam analisadas variáveis distintas nos Cenários 02 e 03 , a priorização destes é pelo método de Classificação. A priorização do Cenário 01 diferencia-se das demais por ser realizada por algoritmo que aborda multicritérios. A hierarquização realizada pelo PROMETHEE II não atendeu a um único objetivo, mas reuniu o melhor resultado para atender às várias óticas incluídas na tomada de decisão.

\section{Conclusões}

Neste trabalho foi proposta a avaliação do Método de Análise Multicriterial PROMETHEE II na priorização para manutenção de pavimentos, a partir da hierarquização de 23 trechos de rodovias diferentes no estado da Paraíba.

Mediante a avaliação de três cenários diferentes, envolvendo critérios técnicos, sociais, econômicos, ambientais e de tráfego, pode-se analisar as variações na hierarquização de acordo com diferentes óticas inseridas nos contextos.

Os resultados da priorização foram distintos quando comparados o Cenário 01 (utilizando critérios técnicos, econômicos, socioambientais e de tráfego), o Cenário 02 (técnico) e o Cenário 03 (relação benefício/custo). Uma das principais razões foi que no Cenário 01 os critérios econômicos, sociais e ambientais representavam $30 \%$ em peso para a tomada de decisão. No Cenário 01 foram considerados critérios que influenciam diretamente na tomada de decisão em construções sustentáveis. Foi capaz de priorizar trechos de rodovias onde as populações tinham maiores necessidades de desenvolvimento social e que pouco interferem nas características ambientais da localidade (PB-073/001 e PB004/007). Os Cenários 02 e 03 restringem-se à classificação de variáveis técnicas ou econômicas.

A utilização do PROMETHEE II tornou possível a avaliação de vários critérios na tomada de decisão, diferentemente dos métodos tradicionais de tomada de decisão em construção rodoviária, que se baseiam em critérios técnicos ou na matemática financeira. A avaliação do Cenário 01 feita pelo tomador de decisão sem utilização de ferramentas de Análise Multicriterial é praticamente inexequível.

A grande vantagem dessa ferramenta é, portanto, a liberdade que o tomador de decisão tem de avaliar vários cenários diferentes, além de inserir diferentes critérios de interesse na análise, o que fornece uma gama maior de opções para a tomada de decisão, inclusive baseadas em critérios relativos à sustentabilidade na construção rodoviária. Além dessa liberdade de análise, outra grande vantagem do Método PROMETHEE II é a utilização de um algoritmo de fácil compreensão e aplicação para o fim de priorização de manutenção de pavimentos rodoviários.

\section{Bibliografia}

ALBUQUERQUE, F. S. Sistema de Gerência de Pavimento para Departamentos de Estradas do Nordeste brasileiro. 2007. 303 f. Tese

(Doutorado em Engenharia Civil) - Escola de Engenharia, Universidade Federal do Rio Grande do Sul, Porto Alegre, 2007.

BRANS, J. P. et al. PROMETHEE: a new family of outranking methods in multicriteria analysis. In: Journal of Operational Research, p. 477-490, North Holland, Amsterdam, 1984.

BRANS, J. P.; VINCKE, P.; MARESCHAL, B. How to Select and How to Rank Projects: the PROMETHEE method. In: European Journal of Operational Research, v. 24, n. 2, p. 228-238, 1986.

\section{DEPARTAMENTO DE ESTRADAS DE} RODAGEM DO ESTADO DA PARAÍBA. Informações Sobre Rodovias do Estado da Paraíba. Disponível em:

<http://www.der.pb.gov.br>. Acesso em: 10 mai. 2006.

\section{HDM-4-b. Analytical Framework \& Model}

Descriptions: part C, economic analysis.

International Study of Highway Development and Management Tools (ISOHDM), The World Bank, Washington, USA, 2000. v. 4.

INSTITUTO BRASILEIRO DE GEOGRAFIA E ESTATÍSTICA. IBGE Cidades. Disponível em: <www.ibge.gov.br>. Acesso em: 11 set. 2007.

INSTITUTO BRASILEIRO DE GEOGRAFIA E ESTATÍSTICA. IBGE Estados@. Disponível em: <www.ibge.gov.br>. Acesso em: 11 set. 2007. 
INSTITUTO BRASILEIRO DE GEOGRAFIA E ESTATÍSTICA. IBGE Mapas. Disponível em: <www.ibge.gov.br>. Acesso em: 11 set. 2007.

IPR-723. Manual de Estudos de Tráfego. Brasília, DF: MT - Departamento Nacional de Infraestrutura de Transportes, Instituto de Pesquisas Rodoviárias, 2006.

LOPES, M. R. C. M. Uso das Metodologias PROMETHEE A F-PROMETHEE na Avaliação de Clientes. 2005. 93 f. Dissertação (Mestrado em Ciência) - Curso de Pós-Graduação em Métodos Numéricos em Engenharia, Universidade Federal do Paraná, Curitiba, 2005.

NHI. Pavement Management Systems. Federal Highway Administration, National Highway Institute Course, 422 p, NHI Course ${ }^{\circ} 131035$, 1998. Disponível em:

<http://www.nhi.fhwa.dot.gov>. 10 jan. 2006.

PORATH, R. Sistema de Gerência de Segurança para o Trânsito Rodoviário: o modelo SGS/TR. 2002. 373 f. Tese (Doutorado em Engenharia de Produção) - Escola de Engenharia, Universidade Federal de Santa Catarina, Florianópolis, 2002.
PORTO, R. La L. Técnicas Quantitativas para o Gerenciamento de Recursos Hídricos. Porto Alegre: Ed. Universidade/UFRGS, 1997.

RÓDENAS, M. del C. E.; BARBERIS, G. F. B. Estudio Comparativo de Métodos de Ayuda a la Decisión Multicriterio en la Valoración y Selección de Alternativas de Inversión. In: JORNADAS DE ECONOMIA Y MATEMATICAS, 10., 2002, Madrid. Anais... Madrid: ASEPUMA, 2002.

\section{SHAHIN, M. Y. Pavement Management for} Airports, Roads and Parking Lots.

Massachusetts: Kluwer Academic Publishers, 1994.

ZUFFO, A. C. Seleção e Aplicação de Métodos Multicriteriais ao Planejamento Ambiental de Recursos Hídricos. 1998. 286 f. Tese (Doutorado em Engenharia Civil) - Escola de Engenharia, Universidade de São Paulo, São Carlos, 1998. 